Erratum

\title{
Erratum to: Non-Existence of Global Solutions For a Quasilinear Benney System
}

S. Antontsev, J. P. Dias, M. Figueira and F. Oliveira

\section{Erratum to: J. Math. Fluid Mech. DOI 10.1007/s00021-009-0014-1}

Theorem 2.1 is not a straightforward variant of Theorem 1.2 in $[1]$, with $\mathbb{R}$ replaced by $\mathbb{R}_{+}$and $H^{1}(\mathbb{R})$ replaced by $H_{o}^{1}\left(\mathbb{R}_{+}\right)$, as referred in the paper, since its correct proof cannot be easily derived from T. Kato's Theorem 6 in [2]. However, all results stated in Theorem 2.1 remain true. Moreover, all results proved in Sect. 3 and 4 apply to any local in time solution $(u, v)$ verifying the properties described in Theorem 2.1. There is also a misprint in formula (4) in Theorem 2.2, where $v^{2}$ must be replaced by $v|u|^{2}$.

\section{References}

[1] Dias, J.P., Figueira, M., Oliveira, F.: Existence of local strong solutions for a quasilinear Benney system. C. R. Acad. Sci. Paris I 344, 493-496 (2007)

[2] Kato, T.: Quasilinear equations of evolution, with applications to partial differential equations. In: Lecture Notes in Mathematics, vol. 448, pp. 335-365. Springer, Heidelberg (1975)

S. Antontsev, J. P. Dias, M. Figueira CMAF/UL and FCUL, Av. Prof. Gama Pinto, 2, 1649-003 Lisbon, Portugal e-mail: anton@ptmat.fc.ul.pt; dias@ptmat.fc.ul.pt; figueira@ptmat.fc.ul.pt

(published online: January 27, 2010)
F. Oliveira

Centro de Matemática e Aplicações FCT-UNL, Monte da Caparica, Portugal e-mail: fso@fct.unl.pt

The online version of the original article can be found under doi:10.1007/s00021-009-0014-1. 